\title{
Cell Type-Specific Roles for Tissue Plasminogen Activator Released by Neurons or Microglia after Excitotoxic Injury
}

\author{
Chia-Jen Siao, ${ }^{1}$ Susana R. Fernandez, ${ }^{2}$ and Stella E. Tsirka ${ }^{1,2}$ \\ ${ }^{1}$ Department of Pharmacological Sciences, Program in Molecular and Cellular Pharmacology, and ${ }^{2}$ Program in Molecular and Cellular Biology, University \\ Medical Center at Stony Brook, Stony Brook, New York 11794-8651
}

Tissue plasminogen activator (tPA) plays important roles in the brain after excitotoxic injury. It is released by both neurons and microglia and mediates neuronal death and microglial activation. Mice lacking tPA are resistant to excitotoxicity and show very limited microglial activation. Activated microglia are neurotoxic in culture, but this phenomenon is not well documented in vivo. To further understand the sequence of events through which tPA mediates microglial activation and neurodegeneration, we have generated mice that exhibit restricted expression of $\mathrm{PA}$ through introduction of tPA transgenes under the control of neuronal- or microglial-specific promoters into tPA-deficient mice. Neither strain of transgenic mice shows abnormal brain morphology or inflammation in the absence of injury, and unilateral intrahippocampal kainate injections into the transgenic mice induced excitotoxicity and microglial activation reminiscent of wild-type mice. However, there are differences in the kinetics of the resulting pathology. The neuronal tPA-expressing mice exhibit accelerated microglial activation compared with wild-type or microglial tPA-expressing mice. However, microglial tPA-expressing mice exhibit greater neurodegeneration. These data suggest a model in which tPA plays different roles after kainate injection depending on whether it is released by neurons or microglia. We propose that $\mathrm{PA}$, initially secreted from injured neurons, acts as a cytokine to activate microglia at the site of injury. These activated microglia then secrete additional tPA, which promotes extracellular matrix degradation, neurodegeneration, and self-proliferation. We suggest that an approach to attenuate microglia-mediated neuronal death in vivo might be to pharmacologically prevent microglial activation.

Key words: cell-cell interactions; microglial activation; transgenic mice; hippocampus; neurodegeneration; in vivo

\section{Introduction}

Excitotoxicity is a key component in many neurodegenerative diseases, including Alzheimer's disease (Mattson et al., 1992), ischemic stroke (Dirnagl et al., 1999), and multiple sclerosis (Pitt et al., 2000). Normally, extracellular levels of glutamate are tightly regulated by brain cells via glutamate transporters (Noda et al., 1999). Excess glutamate released from injured cells upsets this balance and results in hyperdepolarization of neurons causing necrosis and apoptosis (Olney, 1986). Furthermore, injury leads to activation of microglia, the resident immune cells of the CNS.

Reactive microglia play beneficial and detrimental roles in the CNS. Activated microglia present antigen and phagocytose cell debris after injury, thereby preventing damaging molecules from injuring surrounding cells (Banati and Graeber, 1994; Kreutzberg, 1995). After neuronal injury, the cells release chemokines, which diffuse and recruit microglia locally and from other regions of the brain (Blevins and Fedoroff, 1995). Reactive microglia secrete neurotoxic factors as they become activated, including interleukin- $1 \beta$, tumor necrosis factor (TNF)- $\alpha$, nitric oxide (NO), and proteases (Chao and $\mathrm{Hu}, 1994$; McMillian et al.,

Received Sept. 10, 2002; revised Jan. 30, 2003; accepted Feb. 4, 2003.

This work was supported by grants from the National Institutes of Health (NS42168) and the U.S. Army Medical Research and Material Command to S.E.T. We thank Dr. T. Rosenquist and the University Transgenic Facility at Stony Brook for help with generating the transgenic mouse strains. We thank Y. Zhang, J. Sheehan, and Drs. M. Frohman and M. Kritzer for critical reading of this manuscript. S.R.F. is partially supported by a Turner fellowship.

Correspondence should be addressed to Dr. Stella E. Tsirka, Department of Pharmacological Sciences, BST-7, Room 183, University Medical Center at Stony Brook, Stony Brook, NY 11794-8651. E-mail: stella@pharm.sunysb.edu.

Copyright $\odot 2003$ Society for Neuroscience $\quad 0270-6474 / 03 / 233234-09 \$ 15.00 / 0$
1997). We used the protease tissue plasminogen activator (tPA) to study the microglial response to neuronal injury.

tPA is expressed by neurons and microglia in the mouse CNS and, with other extracellular proteases (such as plasmin and thrombin), participates in neurite outgrowth and neuronal development by cleaving proteins of the extracellular matrix and potentially forming a path for extending processes ( $\mathrm{Wu}$ et al., 2000; Jacovina et al., 2001). In addition, tPA is important in modulating the late phase of long-term potentiation (Frey et al., 1996; Baranes et al., 1998).

tPA mediates excitotoxic neurodegeneration by cleaving plasminogen into plasmin and initiating a proteolytic cascade that leads to neuronal death (Tsirka et al., 1995, 1997; Chen and Strickland, 1997). tPA is also involved in microglial activation during excitotoxicity, but this function does not require proteolytic properties of tPA (Rogove et al., 1999). Using a mouse model, we showed that excitotoxic injury is accompanied by full microglial activation, as assayed by microglial morphological changes and protein upregulation $5 \mathrm{~d}$ after kainate (KA) injection (Tsirka et al., 1995). We have also shown in primary cell cultures that microglia, as proposed by Kreutzberg (1996), act as effective sensors of injury (Siao and Tsirka, 2002). Furthermore, previous reports and our cell culture data indicate that $\mathrm{PA}$ released by activated microglia mediates neurodegeneration in a paracrine manner (Rogove and Tsirka, 1998; Flavin et al., 2000; C.-J. Siao and S. E. Tsirka, unpublished observations).

Because tPA is released by both neurons and microglia, the question posed is whether tPA performs multiple roles in vivo, or whether it, as released by different cell types, undertakes distinct 
temporal and functional roles. Addressing this issue is important in understanding how microglia react to injury.

To study the role of tPA in the brain in vivo, we have generated transgenic mice expressing tPA, either in neurons or microglia, in a tPA-deficient $\left(\mathrm{tPA}^{-1-}\right.$ ) background. Here, we characterize these transgenic mice and induce excitotoxicity to examine their neuronal and microglial responses to injury.

\section{Materials and Methods}

All work with animals followed National Institutes of Health guidelines and was approved by the Department of Laboratory Animal Research at the State University of New York (SUNY, Stony Brook, NY). Mice had ad libitum access to food and water and were placed on a $12 \mathrm{hr}$ light/dark schedule.

\section{Generation of transgenic mice}

Microglial tPA construct. Tam 2.5 contains the full-length tPA cDNA, 87 nt of the $5^{\prime}$-untranslated region (UTR), and $746 \mathrm{nt}$ of the $3^{\prime}$-UTR, including the polyadenylation sequence (Rickles et al., 1988). It was linearized using NotI and SmaI. The promoter used to generate microglial tPA-expressing mice was the macrophage colony-stimulating factor (M$\mathrm{CSF}$ (c-fms) protooncogene. It is a monocyte/macrophage-specific promoter that becomes upregulated in acute and chronic injury in the CNS (Akiyama et al., 1994; Raivich et al., 1998). The promoter was released from pSK-cFms (a gift from Dr. Toru Miyazaki, Friedrich Miescher Institute, Basel, Switzerland) using NotI and EcoRV and ligated 5' to the tPA cDNA.

Neuronal tPA construct. Tam2.5 was linearized with SalI and HindIII and inserted 3 ' to the neuronal-specific neurofilament light chain (NF-L) promoter to generate neuron-specific expression. This promoter is most active during embryogenesis, but expression of NF-L promoter-driven transgenes is also detectable after birth (Schlaepfer and Bruce, 1990; Akassoglou et al., 1997). The plasmid containing the promoter was a generous gift from Drs. William Asch and Nisson Schechter (SUNY, Stony Brook, NY).

Generating transgenic mice. NotI and EcoRV (Fms-tPA) or SalI and SmaI (NF-L-tPA) were used to release the promoter-tPA fragments, which were then microinjected into fertilized pronuclei of $\mathrm{tPA}^{-1-}$ mice (on a C57BL/6 background). Five potential founders were generated for each construct; after Southern blotting to confirm genomic integration of transgenes, three lines for each transgene were bred for additional analyses. All of the NF-L-tPA and Fms-tPA lines gave similar results. One line for each construct is described in detail below. Compared with the C57BL/6 and tPA ${ }^{-1-}$ mice, the NF-L-tPA and Fms-tPA strains were equally fertile and produced live young in the expected numbers.

\section{Characterization of transgenic mice}

Tail biopsy and genotyping. Mouse pups at postnatal day 10 (P10)-P15 were tail-clipped, and the genomic DNA was extracted. Briefly, proteinase $\mathrm{K}$ was added to a final concentration of $100 \mu \mathrm{g} / \mathrm{ml}$ in lysis buffer $(100$ м Tris, $\mathrm{pH} 8.5,5 \mathrm{M}$ EDTA, $0.2 \%$ SDS, and $200 \mathrm{M} \mathrm{NaCl}$ ). After incubation at $65^{\circ} \mathrm{C}$ overnight, the resulting suspension was centrifuged at maximal speed for $20 \mathrm{~min}$ at $4^{\circ} \mathrm{C}$. The DNA in the supernatant was precipitated with isopropanol, washed with cold 70\% ethanol, and dissolved in $200 \mu \mathrm{l}$ of $10 \mathrm{~mm}$ Tris $\mathrm{HCl}$ and $1 \mathrm{~mm}$ EDTA, $\mathrm{pH}$ 7.4. One microliter was used for genotyping by PCR using the following primers: Fms-tPA: F1, 5' TAAAAGGGGAAGAAGAGGATCAGC-3'; R1, 5'-GCAGACAAAGTCAGAGAAATACAG-3'; NF-L-tPA: F5, 5'-GATCGATCACAGTCTGCGTCAG-3', and R1. Furthermore, the following internal tPA genotyping primers were used: tPA1, 5'-CCACCTGTGGCCTGAGGCAGTACAA-3'; tPA2, 5'-ATGCCTCATGCTTGCCGTAGCCAGA-3'. In wild-type mice, the $\mathrm{tPA} 1 / 2$ primers produce a $750 \mathrm{bp}$ band, because there are two introns in the genomic sequence between the two primers, whereas in transgenic mice these primers produce a $470 \mathrm{bp}$ band (from the $\mathrm{PAA} \mathrm{cDNA}$ ). To confirm the $\mathrm{tPA}^{-1-}$ background, the neo cassette was amplified using neo $3\left(5^{\prime}\right.$ CTGAATGAACTGCAGGACGA-3') and neo 4 (5'-ATACTTTCTCGGCAGGAGCA-3').

In situ hybridization. Fresh-frozen coronal sections $(20 \mu \mathrm{m})$ were postfixed in $4 \%$ paraformaldehyde, permeabilized with $20 \mu \mathrm{g} / \mathrm{ml}$ pro- teinase K, fixed again in paraformaldehyde, and acetylated. Prehybridization was performed at room temperature (RT) using the following solution: $50 \%$ formamide, $5 \times$ SSC, $5 \times$ Denhardt's solution, $250 \mathrm{gm} / \mathrm{ml}$ baker's yeast mRNA, and $500 \mu \mathrm{g} / \mathrm{ml}$ herring sperm DNA/DEPC distilled water $\left(\mathrm{dH}_{2} \mathrm{O}\right)$. Two of the probes used were generated from linearized pASP, which contains the $3^{\prime}$-UTR of tPA and the T7 promoter generated an antisense probe, whereas the $\mathrm{T} 3$ promoter generated sense probe. Another probe used was generated from the PCR product from tPA1/2, because tPA2 has attached at its $5^{\prime}$ end a T7 promoter. The probes were prepared using the MaxiScript in vitro transcription kit (Ambion, Austin, TX) with digoxygenin (DIG)-labeled UTP according to the manufacturer's instructions and added to the hybridizing sections, which were then incubated at $72^{\circ} \mathrm{C}$ overnight. The anti-DIG antibody (Roche Products, Hertforshire, UK) was used at a 1:2000 dilution, and the secondary alkaline phosphatase-conjugated anti-sheep antibody (Sigma, St. Louis, MO) was used at 1:200. Detection was performed using 5-bromo-4-chloro-3indolyl phosphate/nitroblue tetrazolium (Roche Products) or diaminobenzadine/peroxide ( $\mathrm{DAB} / \mathrm{H}_{2} \mathrm{O}_{2}$; Sigma).

Primary cell culture. Hippocampal neuronal cultures were established as published previously (Siao and Tsirka, 2002). Briefly, embryonic day 17 (E17)-E19 embryos were dissected from killed pregnant mice, and their hippocampi were removed and placed into cold HBSS. After successive trypsin and trypsin-inhibitor incubations, the hippocampi were triturated through a fire-polished pipette. The resulting single-cell suspension was plated onto poly-L-lysine (PLL)-precoated glass coverslips with G3 medium (neurobasal/B27 supplement, $0.5 \mathrm{~mm}$ glutamine, $25 \mu \mathrm{M}$ glutamate, and $10 \mathrm{mg} / \mathrm{l}$ gentamicin). Three to $5 \mathrm{hr}$ later, the medium was completely changed into fresh G3. Three to $5 \mathrm{~d}$ later, the medium was half-changed into G2 (G3 minus glutamate). After $7 \mathrm{~d}$ in culture, neurons were stimulated with $25 \mu \mathrm{m}$ glutamate for $4 \mathrm{hr}$ and then total RNA was extracted as described below.

Microglia were isolated from cultures of mixed cortical cells as described previously (Siao and Tsirka, 2002). Briefly, cortices from E18 or P0 mice were trypsinized and triturated, and the resulting single-cell suspension was plated into PLL-coated $75 \mathrm{~cm}^{2}$ tissue-culture flasks. The medium (DMEM, 10\% FBS, and $40 \mathrm{mg} / 1$ gentamicin) was changed every $3 \mathrm{~d}$. After 10-14 d in culture, the mixed cortical cells establish a confluent layer with bright rounded microglial cells visible on top of the layer. These microglia were removed by $15 \mathrm{~mm}$ lidocaine treatment with gentle shaking; after centrifugation, the pellet was resuspended in complete medium and plated onto PLL-coated glass coverslips. After $2 \mathrm{~d}$ in culture, microglia were stimulated with $100 \mathrm{ng} / \mathrm{ml}$ bacterial lipopolysaccharide (LPS; strain O55:B5; Sigma) for $16 \mathrm{hr}$ and then total RNA was extracted as described below.

Reverse transcriptase PCR. Total RNA was prepared from total brain, cultured primary neuronal, or microglial cells using the Trizol reagent (Invitrogen, San Diego, CA) according to the manufacturer's directions. First strands were synthesized using Superscript II reverse transcriptase (Invitrogen) according to the manufacturer's instructions and using 100 ng of poly-dT primer. Reactions were incubated at $37^{\circ} \mathrm{C}$ for $1 \mathrm{hr}, 42^{\circ} \mathrm{C}$ for $30 \mathrm{~min}$, and $72^{\circ} \mathrm{C}$ for $10 \mathrm{~min}$. PCR was performed using $1 \mu \mathrm{l}$ of the reverse transcriptase reaction product. The PCR products were separated on a $1 \%$ agarose gel and stained with ethidium bromide. To ascertain cDNA quality, $\beta$-actin was amplified. In addition to the tPA1/2 or transgenic tPA primers used above, the following additional primers were used: F4/80, 5'-GACGGTTGAGCAGACAGTGA-3' and 5'-TGCTAGTGGAGGCAGTGATG-3'; BDNF, 5' -CCTGGATGCCGCAAACATG-3' and 5' CCACTATCTTCCCCTTTTAATGG-3'; actin, 5' -GGCCACTGCCGCATCCTCTT-3' and 5'-AGAGCCTCAGGGCATCGGAAC-3'.

\section{In vivo studies}

Induction of excitotoxicity. For intrahippocampal injections, adult mice were injected intraperitoneally with atropine $(0.6 \mathrm{mg} / \mathrm{kg})$, anesthetized with $2.5 \%$ avertin $(0.02 \mathrm{ml} / \mathrm{gm})$, and $0.75 \mathrm{nmol} \mathrm{KA}$ (Alexis Biochemicals, San Diego, CA) in $300 \mathrm{nl}$ (or the same volume of PBS control, or 0.25 $\mathrm{nmol}$ of $\mathrm{KA}$ as a low dose) was injected into the following coordinates from bregma: $-2.5 \mathrm{~mm}$ anteroposterior, $1.7 \mathrm{~mm}$ mediolateral, and -1.6 $\mathrm{mm}$ dorsoventral. The injection lasted for $1 \mathrm{~min}$, and the needle was left in place for an additional 2 min to prevent reflux. Mice were killed at 
various indicated times after injection, and their brains were removed. Each brain was first trimmed so that some tissue from both the injected and the noninjected sides could be frozen for RNA purification and protein extraction. The remainder of the brain was frozen on dry ice in optimal cutting temperature compound (Tissue-Tek; Miles, Elkhart, IN). All of the NF-L-tPA and Fms-tPA lines gave similar results. One line for each construct is described in detail below.

Cresyl violet staining. To detect neuronal survival/death, fresh-frozen sections were postfixed in $4 \%$ paraformaldehyde and then dipped in cresyl violet dye ( $2.5 \mathrm{gm}$ of cresyl violet, 30 $\mathrm{ml}$ of $1 \mathrm{M}$ sodium acetate, $170 \mathrm{ml}$ of $1 \mathrm{M}$ acetic acid, and $300 \mathrm{ml}$ of $\mathrm{dH}_{2} \mathrm{O}$ ) for 5-10 min. Sections were serially dehydrated in graded ethanol and then defatted in xylenes. Finally, sections were coverslipped with Permount (Fisher Scientific, Houston, TX). The stained sections were photographed using a digital camera (Nikon CoolPix 990; Nikon, Tokyo, Japan) on a Nikon Eclipse TS100 microscope under brightfield optics and then converted into an uncompressed tagged image file using Adobe Photoshop (Adobe Systems, San Jose, CA). The degree of neurodegeneration was measured using the freeware Scion Image beta 4.02 (Scion, Frederick, MD), downloadable at http://www.scioncorp.com/index.htm. Briefly, the TIF file was loaded into Scion Image, and the freehand tool was used to define the length of pyramidal neuronal loss and measured as arbitrary units. The entire length of the hippocampal pyramidal layer was measured in the same manner, and the percentage loss of neurons on the injected side was calculated. A minimum of five sections was quantified for each time point per genotype.

Immunohistochemistry. Fresh-frozen coronal sections $(20 \mu \mathrm{m})$ were cut using a Leica (Nussloch, Germany) cryostat, mounted onto slides (Fisher SuperFrost Plus; Fisher Scientific), air dried, and postfixed in $4 \%$ paraformaldehyde/PBS. Endogenous peroxidase activity was quenched with peroxide treatment $\left(3 \% \mathrm{H}_{2} \mathrm{O}_{2}\right.$ in $\left.\mathrm{PBS}, 30 \mathrm{~min}\right)$. After blocking in serum of the host of the secondary antibody [ $5 \%$ serum in PBS-T $(0.5 \%$ Triton X-100 in PBS)], the primary antibody was added (into 5\% serum in PBS-T) at the following dilutions: 1:1000 50.1 tPA (generated inhouse); 1:200 F4/80 (microglia/monocytes; Serotec, Indianapolis, IN); 1:500 5D4 [activated microglia in brain (Jander and Stoll, 1996; Bertolottoa et al., 1998), activated microglia, macrophages, and precursors of oligodendrocytes in the spinal cord (Jones and Tuszynski, 2002); Seikagaku Kogyo, Tokyo, Japan]; 1:500 neuronal-specific nuclear protein (neuronal nuclei; Chemicon, Temecula, CA). Sections were incubated in primary antibody overnight at $4^{\circ} \mathrm{C}$. After a $10 \mathrm{~min}$ wash in PBS, appropriate biotinylated secondary antibodies were added in serum/PBS-T and incubated for $30 \mathrm{~min}$. After washing in PBS for $10 \mathrm{~min}$, the ABC reagent was added to conjugate avidin-peroxidase to the immune complex (Vector Laboratories, Burlingame, CA) according to manufacturer's directions for $30 \mathrm{~min}$. The blot was washed for $10 \mathrm{~min}$, and the signal was visualized using $\mathrm{DAB} / \mathrm{H}_{2} \mathrm{O}_{2}$. Sections were successively dehydrated in ethanol, defatted in xylenes, and coverslipped with Permount (Fisher Scientific). To enhance the DAB signal, a silver/gold method was used before dehydration (Kritzer, 2000). Briefly, sections were incubated in $1 \%$ silver nitrate, $\mathrm{pH} 7.0$, at $55^{\circ} \mathrm{C}$ in the dark for $50 \mathrm{~min}$, rinsed in $\mathrm{dH}_{2} \mathrm{O}$, incubated in $0.2 \%$ gold chloride in the dark for $15 \mathrm{~min}$, rinsed again in $\mathrm{dH}_{2} \mathrm{O}$, and then incubated in $5 \%$ sodium thiosulfate for $10 \mathrm{~min}$. Slides were dehydrated and mounted as described above. To perform semiquantitative analysis, the slides were treated at the same time with the same reagents, and different fields of view at the same magnification were photographed and digitized as described above. To quantify cells, we counted the number of nuclei whose cell bodies were stained with 5D4. b
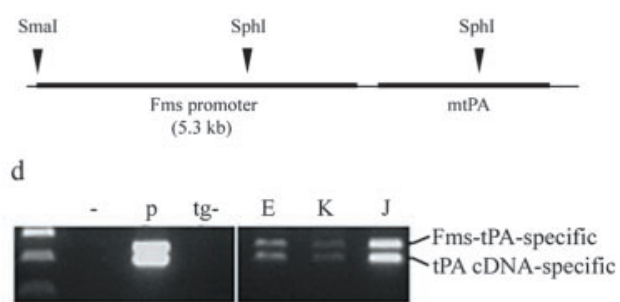

f

NF-L-tPA

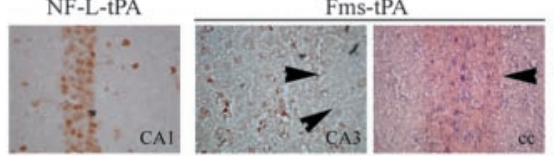

\section{.}

d
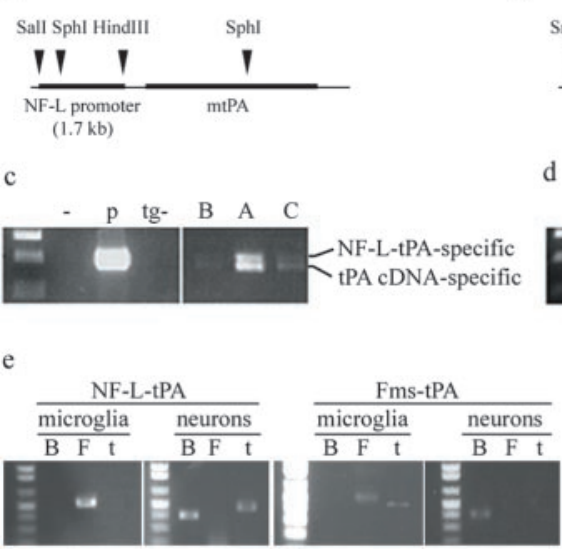

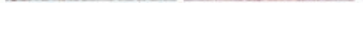

nstruct. The promoter for the neurofilament light chain gene was fused to $\mathrm{mtPA}$, and relevant restriction enzyme sites are labeled. $b$, A microglia-specific transgenic construct. The promoter for the gene encoding the receptor for M-CSF (also known as c-fms) was fused to mouse PA cDNA. c, Genotyping PCR

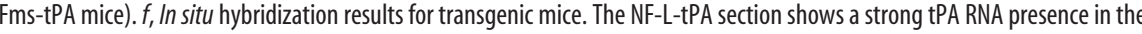
pyramidal neurons. The Fms-tPA sections show a more diffuse tPA RNA presence around the CA3 subfield (arrowheads) as well as in the CC (arrowheads). Original magnification, $100 \times$.

We then used the freehand shape tool in the Image software to delineate the microglial cell and subsequently measured the area that is taken up by the cell as pixels squared. We present this data as relative measurements and for comparisons across groups.

In situ zymography. Fresh-frozen coronal sections $(20 \mu \mathrm{m})$ were overlaid with a mixture consisting of $1 \%$ low-melting agarose, $1 \mathrm{mg} / \mathrm{ml}$ plasminogen, amiloride [which inhibits urokinase (uPA)], $0.1 \mathrm{~mm}$ Tris-Cl, $\mathrm{pH} 7.5$, and nonfat dry milk. A coverslip was added to flatten the overlay, and the sections were incubated at $37^{\circ} \mathrm{C}$ in a humidified chamber. Activity of tPA is visualized where there is degradation of casein (a clearing of the white matrix) by the activation of plasminogen into plasmin.

Amidolytic assay. Brain lysate from wild-type and both transgenic strains (all transgenic lines) of mice were homogenized with $0.25 \%$ Triton X-100, and cell debris was centrifuged and removed. The supernatant $(30 \mu \mathrm{l})$ was added to microplate wells containing $170 \mu \mathrm{l}$ of the master

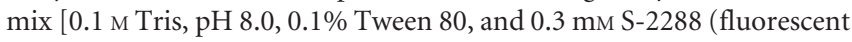
amidolytic substrate; Chromogenix, Milano, Italy)]. Absorbance (405 $\mathrm{nm}$ ) was read at $t=0$ and $3 \mathrm{hr}$. Relative tPA activity was measured using recombinant tPA protein as a control at the same time point.

Statistics. In all quantification procedures, $n$ refers to the number of animals under each condition. The observers were blinded to the nature of the experimental manipulation. Statistical analysis was performed using a two-tailed $t$ test; $p<0.05$ is considered significant. Experiments were performed in duplicate or triplicate.

\section{Results}

Cell type-specific expression of tPA in transgenic mice.

Transgenic mice that express tPA only from neurons or microglia were generated in a tPA-deficient mouse strain. For neuronspecific tPA expression, the murine tPA cDNA (mtPA) was fused to the promoter of the NF-L gene. Figure 1 shows the transgenic construct schematic $(a)$ and representative genotyping PCR results from tail-biopsy DNA (c). tPA genomic DNA gives rise to a 750 bp PCR product, whereas the introduced cDNA generates a $470 \mathrm{bp}$ band. Similar results were observed for three independent lines (A-C). Results for line A will be shown in this study. 


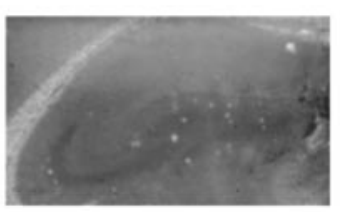

C57

c

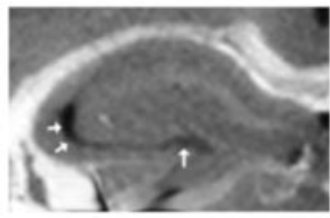

NF-L-tPA

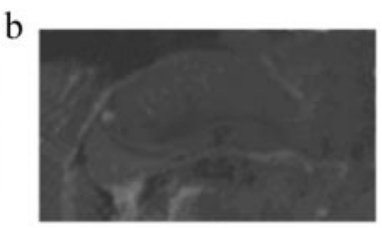

$\mathrm{tPA}^{-/-}$

d

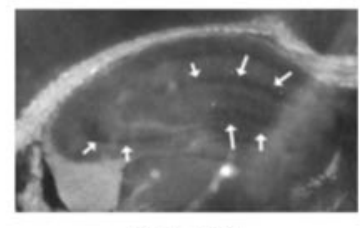

Fms-tPA
Figure 2. tPA secreted by transgenic mice is active. An in situ zymographic assay was used on sections from wild-type $(a), \mathrm{PPA}^{-1-}(b)$, NF-L-tPA $(c)$, and Fms-tPA $(d)$ mice to visualize endogenous $\mathrm{PAA}$ proteolytic activity. All sections except for $\mathrm{PAA}^{-1-}$ showed tPA activity in the CA3/hilus regions of the hippocampus (arrows). The arrows in $d$ also indicate the stratum radiatum. Endogenous uPA activity was inhibited with amiloride. Original magnification, 10X.

tPA mRNA and protein are present at high levels in different regions of the brain, including the hippocampus, amygdala, and cerebellum, and are expressed by both neurons and microglia (Sappino et al., 1993; Seeds et al., 1995; Tsirka et al., 1997). To express tPA in monocytes/macrophages, we used the promoter for the receptor for M-CSF, also known as the protooncogene c-fms. Figure 1 shows the transgenic construct schematic $(b)$ and representative genotyping PCR results $(d)$. Similar results were observed for three independent lines (E, J, K); data for line E are shown below.

Reverse transcriptase PCR (Fig. 1e) and in situ hybridization (Fig. $1 f$ ) analyses were used to determine whether the transgenes are expressed in the intended cell types. Purified primary neurons or microglia from wild-type, $\mathrm{tPA}^{-1-}$, NF-L-tPA, and Fms-tPA mice were prepared. Microglia were primed with $100 \mathrm{ng} / \mathrm{ml}$ LPS for $16 \mathrm{hr}$, and neurons were stimulated with $25 \mu \mathrm{M}$ glutamate for $3.5 \mathrm{hr}$. RNA was then extracted and reverse transcribed, and the cDNA was subjected to PCR using primers for tPA, BDNF (for neurons), and $\mathrm{F} 4 / 80$ (for microglia). As shown in Figure 1e, the NF-L-tPA mice expressed tPA in neurons but not in microglia, whereas the Fms-tPA mice expressed tPA only in microglia. In situ hybridization on transgenic brain sections (Fig. $1 f$ ) demonstrated an intense tPA signal over NF-L-tPA neurons in the CA1 subfield. In the Fms-tPA mice, the tPA signal was distributed in a punctate pattern across the hippocampus, consistent with the distribution of microglia, with particularly strong staining in the corpus callosum (CC). In addition, the Fms-tPA signal colocalized with cells that stain with the antibody F4/80, which is specific for macrophages and microglia (data not shown). No signal was observed in the $\mathrm{tPA}^{-/-}$mice (data not shown). These results confirm that the constructs were expressed in the restricted manner intended by the choice of promoters.

\section{Transgenic mice express active tPA protein}

To confirm that the transgenic mice expressed tPA specifically in the hippocampus, we performed in situ zymographic analyses on sections of wild-type, $\mathrm{tPA}^{-1-}$, and transgenic mouse brains. Figure $2 a$ shows that in wild-type mice, tPA activity localizes to the CA3 and the hilus of the hippocampus (dark areas, arrows) (Sappino et al., 1993; Tsirka et al., 1995; Sallés and Strickland, 2002). No plasminogen activator activity was detected in the $\mathrm{tPA}^{-/-}$ sections (Fig. 2b). tPA activity localized in NF-L-tPA mice in a pattern similar to what had been observed for endogenous tPA in wild-type mice (Fig. 2c). Although this result may seem unexpected given that the NF-L promoter should be driving expression in all neuronal cells, it is actually consistent with findings from wild-type mice: tPA mRNA is localized in all neuronal pyramidal subfields in the hippocampus (Tsirka et al., 1997), but protein activity is only detected in the CA3 and hilus, possibly because of the presence elsewhere of protease inhibitors such as plasminogen activator inhibitor-1 and neuroserpin (Cuzner et al., 1996; Hastings et al., 1997; Krueger et al., 1997). Robust tPA activity in Fms-tPA mice was observed in the stratum radiatum (Fig. $2 d$, arrows) as well as in the CA3 subfield.

To quantitate the amount of tPA synthesized by the transgenic mice under basal conditions, we used the amidolytic assay to compare the activity of tPA expressed in protein extracts from brains of the transgenic and wild-type animals. We found that the transgenic mice express lower amounts of tPA than do the wildtype animals (which physiologically express both neuronal and microglial tPA): $0.7 \mu \mathrm{g} / \mathrm{mg}$ for C57BL/6, $0.5 \mu \mathrm{g} / \mathrm{mg}$ for NF-LtPA, $0.3 \mu \mathrm{g} / \mathrm{mg}$ for Fms-tPA. KA-injected transgenic mice expressed higher amounts of tPA. For mice from both the neuronal and microglial genotypes, the amidolytic assay reported $1.5 \mu \mathrm{g}$ $\mathrm{tPA} / \mathrm{mg}$ total protein; this represents a threefold increase in active tPA in NF-L-tPA mice and a fivefold increase in active tPA in Fms-tPA mice ( $n=2$ for each genotype in each condition).

\section{Neurons and microglia from uninjured transgenic mice appear normal}

tPA expression is tightly regulated in the CNS under basal conditions. Therefore, the constitutive expression of transgenic tPA under the selected promoters could sensitize neurons and microglia to injury and degeneration even in the basal state. However, we examined naive uninjured mice and did not observe spontaneously degenerating neurons or constitutively active microglia (Fig. $3 a-d$ ). We also injected transgenic mice unilaterally with PBS. In all genotypes (wild-type, tPA ${ }^{-1-}$, NF-L-tPA, and FmstPA), the injury caused by the injection did not induce neurodegeneration. Furthermore, parenchymal microglia did not become activated (Fig. $3 e-h$ ) (data not shown for wild-type and $\mathrm{tPA}^{-1-}$ mice), indicating that low constitutive expression of tPA does not suffice to sensitize the cells even to mechanical injury. These results also demonstrate a lack of activated microglia or infiltrating macrophages, indicating that the blood-brain barrier (BBB) is not compromised and parenchymal tissue is not inflamed.

\section{Kainic acid induces neurodegeneration in transgenic mice}

We injected $25 \mathrm{nmol}$ of KA unilaterally into the hippocampus of C57BL/ 6 animals and both strains of transgenic mice (all transgenic lines) and examined neuronal survival at several times from $6 \mathrm{hr}$ to $5 \mathrm{~d}$ after injury using cresyl violet staining (Fig. $4 A$ ). The left panel shows representative wild-type (C57BL/6) sections as a reference, whereas the middle and right panels show NF-L-tPA (line A) and Fms-tPA (line E) sections, respectively. Results from other lines, although not performed in numbers equal to those described and quantified for lines A and E, suggest that they react in a similar manner to this dose of KA (Fig. $4 B$ ). The quantification of neuronal survival is summarized in Figure 5 as the percentage of neuronal death, and relevant measurements from wild-type mice are included as a reference. Interestingly, the NFL-tPA mice exhibited a lower degree of neurodegeneration at $1 \mathrm{~d}$ after injection of the excitotoxin $(29.3 \%)$ compared with the 


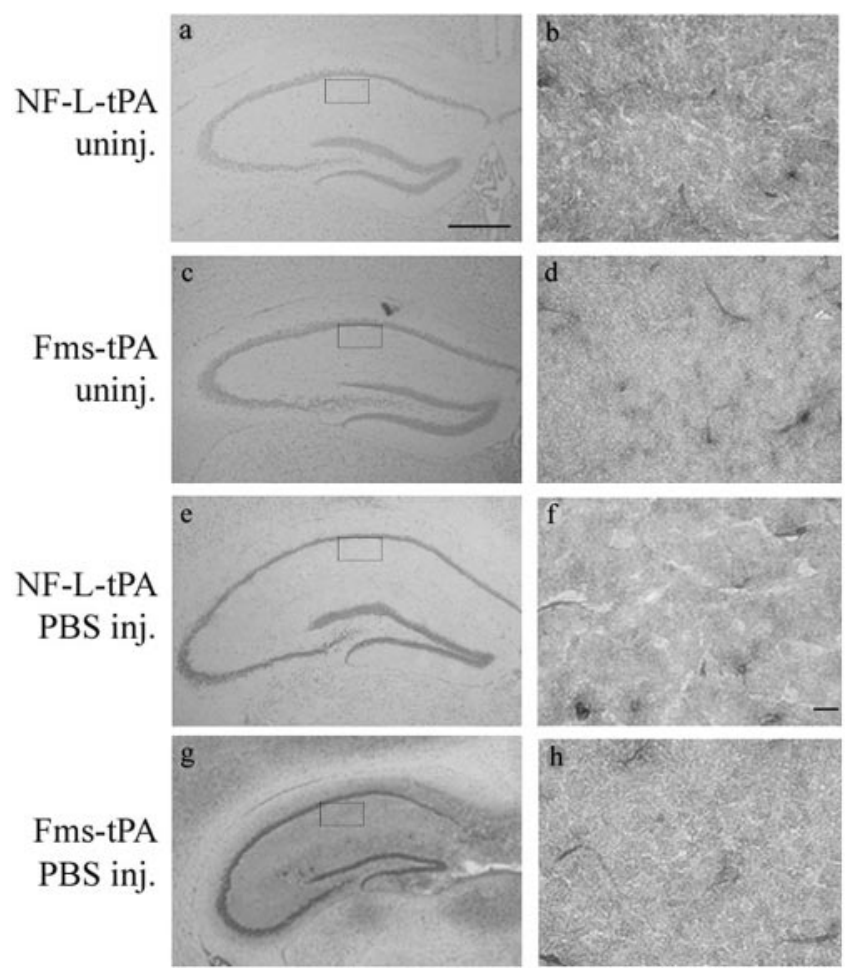

Figure 3. Transgenic mice show no neuronal death or microglial activation without injury. Cresyl violet stained hippocampal sections show an intact $C A$ pyramidal neuronal layer in mice that were untreated $(a, c)$ or injected with PBS only $(e, g) \cdot b, d, f, h$, In addition, sections (boxed insets) from the same mice were immunodetected with $F 4 / 80$ antibody, which recognizes microglia/macrophages; the lack of specific staining shows that the brain parenchyma is not injured. Dorsal is at the top of all panels. Original magnifications: cresyl violet panels, 20X; immunohistochemistry panels, $400 \times$. The high-magnification panels are from the CA1 subfield of the hippocampus. uninj., Uninjected; inj., injected.

Fms-tPA (62.2\%) and wild-type (59.8\%) mice. NF-L-tPA mice also exhibited a slower rate of neurodegeneration compared with the Fms-tPA mice (Fig. 5, bottom panel). Both strains of transgenic mice showed a similar extent of neuronal death by day 5 (67.9 and 69.9\%), although neither appeared to be as high as that of the wild-type mice (86.4\%). In control experiments, a low dose of KA ( $0.25 \mathrm{nmol})$ was injected into these mice. As in wild-type mice, negligible neuronal death was observed after this milder injury (data not shown), suggesting that these transgenic mice can be compared directly with their wild-type or $\mathrm{tPA}^{-/-}$genetic background controls.

The difference in the time of neuronal degeneration between NF-L-tPA and Fms-tPA neurons in their response to the normal dose of KA suggested either that the neuronal tPA transgene does not synthesize enough tPA to initiate the extracellular proteolytic cascade necessary for neuronal death, or that the initial tPA release from injured neurons serves a role in addition to that of cleaving plasminogen to plasmin. To address the first possibility, we injected tPA $(120 \mathrm{ng} / \mathrm{ml})$ into the transgenic mice in conjunction with KA and then examined whether they responded to the KA insult with a restored (i.e., wild-type) rate and degree of neurodegeneration $(n=2$ mice per time point per transgenic strain; numbers given are the mean percentage of neuronal loss). With $120 \mathrm{ng} / \mathrm{ml}$ tPA, NF-L-tPA mice exhibited increased neuronal death at $6(10.2 \%)$ and $12(40.2 \%) \mathrm{hr}$, consistent with an acute role of tPA in cleaving plasminogen and mediating anoikis [derived from the Greek word for "homelessness," a term used to describe apoptosis that is induced when cells lose integrinmediated contacts with the extracellular matrix (Cardone et al., 1997)], leading to cell death. There was no decrease in survival with a lower dose of tPA $(12 \mathrm{ng} / \mathrm{ml}) ; 56 \%$ of the cells died by $1 \mathrm{~d}$ after injection. Coinjection of tPA $(120 \mathrm{ng} / \mathrm{ml})$ and KA into the Fms-tPA mice resulted in exacerbation of early neuronal death: $43.5 \%$ at $6 \mathrm{hr}$ and $51.5 \%$ at $12 \mathrm{hr}$ after injection. These observations are in agreement with previous data that showed that increased extracellular levels of tPA are directly responsible for neuronal death and suggest that the microglial component is very important in mediating the early acute neurodegeneration after excitotoxic injury. Therefore, it appears that the lower amount of tPA from the NF-L transgene caused the decreased rate of neuronal death in the NF-L-tPA mice. However, because activated microglia may also play an important, although not well characterized, role in neurodegeneration, we also examined this cellular response to the KA injection.

\section{Excitotoxicity induces microglial activation in transgenic mice}

The progress of microglial activation was followed during the same time points as neuronal death, using the 5D4 and F4/80 antibodies (Fig. 6). 5D4, which recognizes activated microglia, was used for earlier time points (6 hr to $1 \mathrm{~d}$ ) and F4/80, which stains mature macrophages and microglia, was used for the $2-5 \mathrm{~d}$ time points. The $5 \mathrm{~d}$ time point was chosen because it represents in the KA-injection excitotoxicity model the earliest time point at which maximal morphologically visible microglial activation is observed (Andersson et al., 1991). Despite the lesser degree of neurodegeneration, the NF-L-tPA mice exhibited 5D4-staining microglia beginning $12 \mathrm{hr}$ after KA injection, compared with wild-type and the microglial tPA-expressing mice, which exhibited activated microglia only subsequently at $2 \mathrm{~d}$ after injury. These 5D4-staining cells are not macrophages that infiltrated the brain parenchyma, because the F4/80 antibody, which recognizes all cells of the macrophage/monocyte lineage (Lawson et al., 1990), did not stain cells at the earlier time points (data not shown). It was reported recently that 5D4 also stains precursors of oligodendrocytes (OPCs) in the rat spinal cord (Jones and Tuszynski, 2002). We fluorescently doubly stained brain sections for the established marker of OPCs, the chondroitin sulfate proteoglycan NG2, and 5D4 for microglia. No obvious colocalization of the two fluorophores was observed by confocal microscopy, possibly because we are using different fixation and staining protocols.

Using a DAB enhancement method, we semiquantitatively measured the amount of microglial activation (Table 1) using cell counts (nuclei present per field of view per section; middle column) and cell size (area covered by each microglial cell in pixels squared; right column). Because very few cells are visible by 5D4 staining at $12 \mathrm{hr}$ for the wild-type and Fms-tPA sections, this time point was omitted from analysis. As Table 1 shows, more cells accumulated in wild-type sections compared with transgenic mice by $3 \mathrm{~d}$ after KA injury, despite the fact that at $1 \mathrm{~d}$ after injury, no activated microglia were detectable. The average number found per field of view by $3 \mathrm{~d}$ was higher in wild-type sections than in either transgenic strain. The NF-L-tPA microglia were activated to a greater extent earlier, as Figure 6 shows pictorially. The size of these visible activating microglia also increased for all genotypes over the first $3 \mathrm{~d}$ after injury (Table 1 , area in $\mathrm{px}^{2}$ ). NF-L-tPA microglia occupy a larger area and appear more activated in morphology compared with wild-type or Fms-tPA mice at $1 \mathrm{~d}$. 
A wt

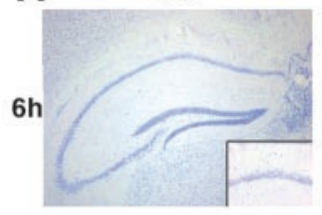

$12 \mathrm{~h}$
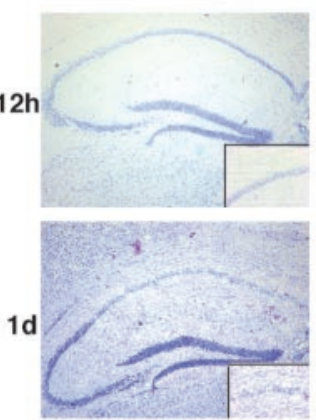

NF-L-tPA
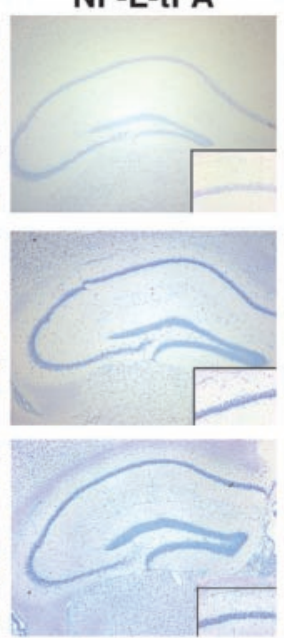

Fms-tPA
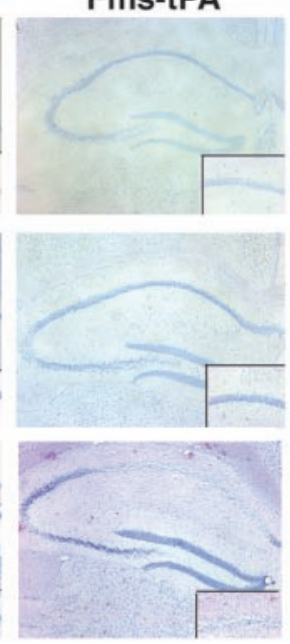

$5 d$

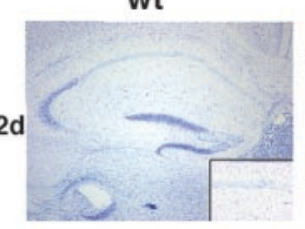

$3 d$
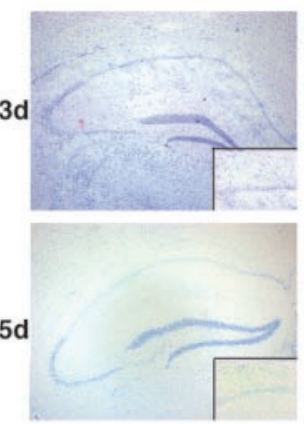

NF-L-tPA
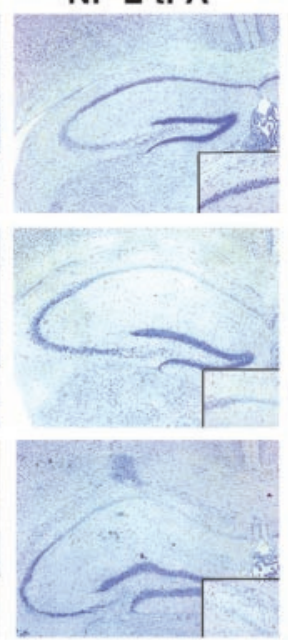

Fms-tPA
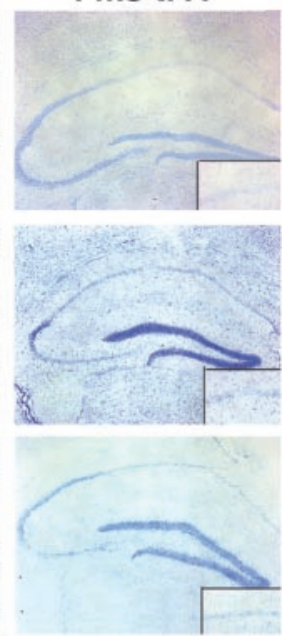

B
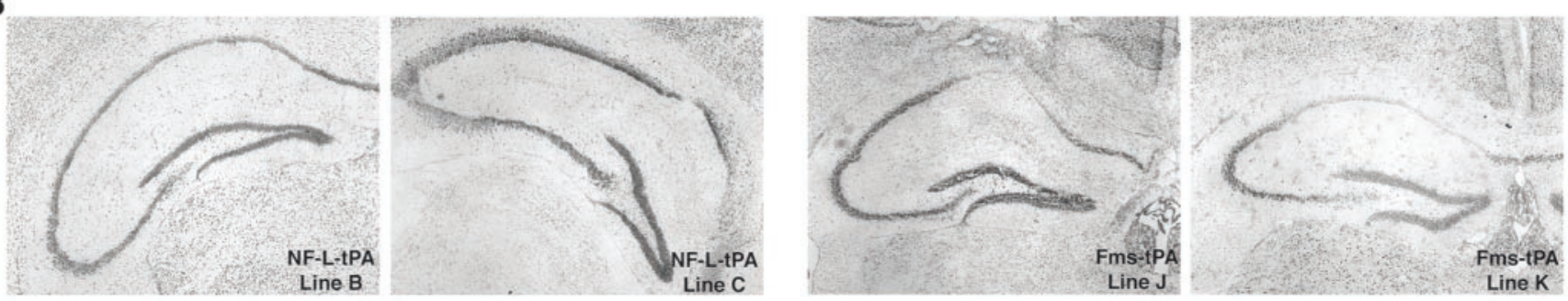

Figure 4. Characterization of neuronal death in wild-type and transgenic mice after excitotoxicity. Mice were injected with KA and then killed at the indicated time points. Neuronal death was determined using cresyl violet, which outlines an intact pyramidal neuronal layer. Lack of staining indicates that the cells are dead. $A$, Left column, wild-type (wt) sections; middle column, NF-L-tPA sections; right column, Fms-tPA sections. Dorsal is at the top of all panels. Insets, Higher magnification of the CA1 subfield showing shrunken soma of dead neurons compared with larger stronger-staining cell bodies of neurons that survived the KA injection. Original magnification: large panels, $10 \times$; insets, $200 \times$. The hippocampal measurements were performed by investigators blind to the genotypes of the mice as well as to the time points after KA injection for each sample. $B$, A response comparable with that seen for KA injection is observed in the other transgenic lines as well at all time points. The sections presented are from $1 \mathrm{~d}$ after KA injection.

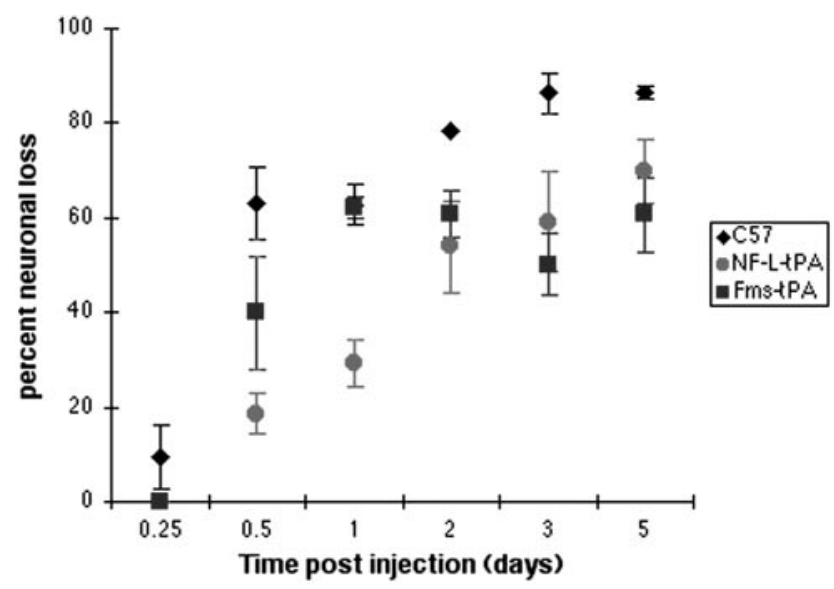

Figure 5. Quantification of neuronal death after KA injection. Sections treated as in Figure 4 were grouped by time points. The amount of neurodegeneration was measured using Scion Image and presented as the percentage of total hippocampal length of the ipsilateral injected side (SEM). These data are graphically presented as well. Note that the NF-L-tPA mice show a slower increase of neuronal loss at early time points (up to $2 \mathrm{~d}$ ) compared with wild-type or Fms-tPA mice, although they match the percentage lost by Fms-tPA mice by $5 \mathrm{~d}$.

When NF-L-tPA mice were coinjected with tPA and KA, microglia were visible as ramified cells by $12 \mathrm{hr}$ after injury, although their morphological changes were not complete (data not shown). This upregulation of the 5D4 epitope occurred at the same time as in NF-L-tPA mice injected with KA alone (Fig. 6).
The ramified microglia were not visible at $6 \mathrm{hr}$ after KA injection, suggesting that although morphologically these $(12 \mathrm{hr}$ ) ramified microglia are not activated, they are upregulating certain proteins (such as the 5D4 keratan sulfate epitope) in response to the injury by $12 \mathrm{hr}$ after excitotoxic injury. Furthermore, the extracellular levels of tPA in the NF-L-tPA CNS parenchyma are enough to mediate microglial activation and cell-cell signaling.

Semiquantification of the number of microglia in NF-L-tPA mice at $12 \mathrm{hr}$ after either KA alone (10 per field of view) or with tPA plus KA coinjection (15-16 per field) suggested that increasing extracellular tPA concentration can either recruit more microglia or induce proliferation among microglia already present at the injury site (data not shown). The Fms-tPA mice coinjected with tPA and KA also show a general upregulation of microglial staining using 5D4 by $12 \mathrm{hr}$ (to 15 cells per field), whereas such upregulation was not observed when the mice were injected with KA alone (Fig. 6).

Perineuronal cells appeared to activate later than more distal microglia that migrate toward the site of neuronal injury from the corpus callosum or stratum radiatum (NF-L-tPA) (Fig. 6, 1 d). These perineuronal cells are not astrocytes or oligodendrocyte precursor cells, because they did not colocalize with GFAP or NG2 antibodies, respectively (data not shown).

\section{Discussion}

Microglial activation is a contributor to neuronal death in a variety of neurodegenerative diseases, and a recent push aims at ways to decrease or inhibit it. Microglia receive and send signals 

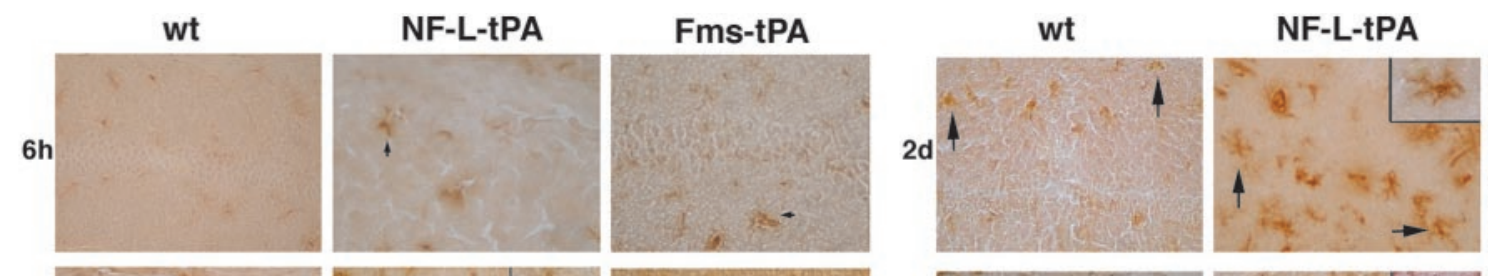

Fms-tPA
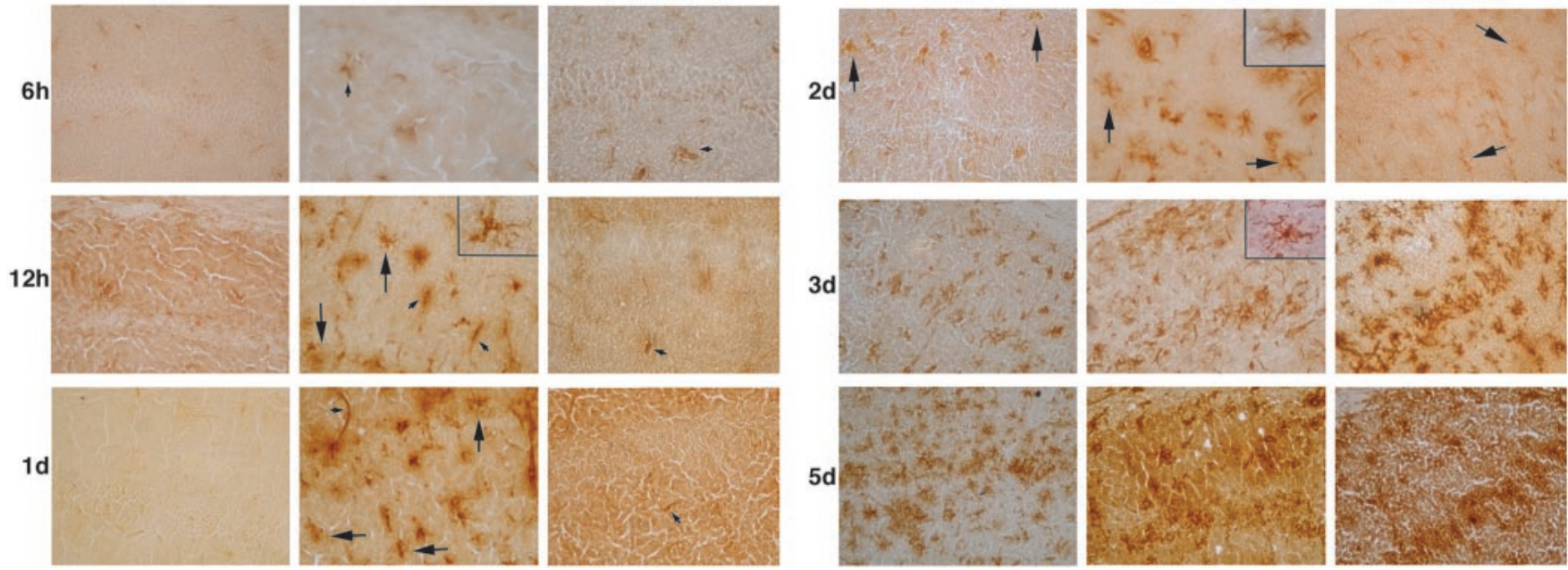

Figure 6. Characterization of microglial activation in wild-type (wt) and transgenic mice after excitotoxicity. Mice were processed as described in Materials and Methods and Figure 4. Sections were immunodetected using either $5 \mathrm{D} 4(6 \mathrm{hr}$ to $1 \mathrm{~d}$ ) or $\mathrm{F} 4 / 80(2-5 \mathrm{~d})$ antibodies. Note that even by $12 \mathrm{hr}$ some activated microglia are visible, although not in the proximity of dying neurons in the CA1 field. Both antibodies recognize antigen in the microvasculature (small arrows), but activated microglia are clearly defined (large arrows, insets). Left column, Wild-type sections; middle column, NF-L-tPA sections; right column, Fms-tPA sections. Dorsal is located at the top of each panel. Original magnification: large panels, 200X; insets, 400X.

Table 1. Evaluation of microglial activation

\begin{tabular}{|c|c|c|}
\hline & Average number of cells & Cell size [area $\left.\left(\mathrm{px}^{2}\right)\right]$ \\
\hline \multicolumn{3}{|c|}{ (57BL/6 } \\
\hline $1 \mathrm{~d}$ & 0 & 0 \\
\hline $2 d$ & 23.7 & 734.0 \\
\hline $3 d$ & 41.3 & 1021.4 \\
\hline \multicolumn{3}{|l|}{ NF-L } \\
\hline $1 d$ & 11.0 & 849.2 \\
\hline $2 d$ & 28.0 & 1241.1 \\
\hline $3 d$ & 31.7 & 1459.7 \\
\hline \multicolumn{3}{|l|}{ Fms } \\
\hline $1 d$ & 4.0 & 0 \\
\hline $2 d$ & 19.3 & 762.7 \\
\hline $3 d$ & 32.0 & 640.8 \\
\hline
\end{tabular}

5D4- or F4/80-stained sections were detected using DAB/peroxide and enhanced with silver/gold as described in Materials and Methods. Only sections from 1, 2, and 3 d after KA injection are included for measurement. The number of cells (nuclei) on each section was counted. Using Scion Image, the shape of detectable microglia was outlined, and the average area enclosed by the outline was determined as the square of the number of pixels. At least three fields

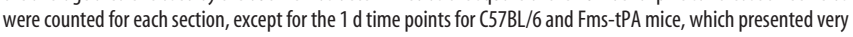
few countable cells; therefore, for these mice only one field was counted for each genotype.

to neurons during trauma and disease. tPA links these cell types, because it is expressed by both and mediates neuronal death and microglial activation. Here, we used an experimental model of neuronal death, that of intrahippocampal excitotoxin injection. Although experimental excitotoxicity does not necessarily represent the clinical aspects of neurodegenerative diseases, it does provide a means to dissect the mechanism by which cell death progresses, and it is excitotoxicity that has been hypothesized to contain the mechanisms by which several neurodegenerative diseases develop.

Transgenic mice expressing active tPA from either neurons or microglia on a $\mathrm{PA}^{-/-}$background were produced and characterized. The expression pattern of tPA activity differs slightly between the two strains of mice: in addition to a weak CA3/hilar localization, Fms-tPA mice also express active tPA in the stratum radiatum, consistent with observations that in wild-type mice, many activated microglia arise from this region and migrate toward the injured neurons in the CA1-CA3 subfields.

One concern with these transgenic mice is the constitutive promoters used. The zymography (Fig. 2) and amidolytic assay results suggest that neither strain of transgenic mice expresses tPA at levels higher than wild-type mice, yet higher than normal levels of tPA by itself do not appear to be detrimental to mice (Madani et al., 1999). The available tPA protein extracellularly is very low without stimulation, because tPA is normally sequestered in vesicles in neurons, and chemical depolarization by potassium causes its release (Gualandris et al., 1996; Parmer et al., 1997). Similar sequestration could be possible in microglia, but the subcellular localization of tPA in resting or activated microglia has not been determined.

We characterized the response of the transgenic mice to excitotoxicity by examining neuronal survival and microglial activation after KA injection. Both strains of transgenic mice respond to KA injury in a manner similar to that seen for wild-type mice: a high percentage of CA1 and CA3 pyramidal neurons die, and the microglia are fully activated (as evidenced by morphological and immunohistochemical analyses) by $5 \mathrm{~d}$ after the injection.

Two differences were evident among these transgenic mice: first, a slower progression of neuronal death with the NF-L-tPA mice, and second, an earlier microglial reaction to the injury in NF-L-tPA mice, when compared with Fms-tPA and wild-type mice. The first difference (slower progression of neuronal death) would suggest that the amount of tPA made and released by the NF-L-tPA neurons is insufficient to promote plasminogen cleavage and mediate the initial neuronal death. However, this may not be the case, because infusion of a low concentration of tPA $(12 \mathrm{ng} / \mathrm{ml})$ into $\mathrm{PA}^{-1-}$ mice before $\mathrm{KA}$ injection did not result in a linear reduction of the extent of neurodegeneration compared with that observed when wild-type mice were injected with KA (or when $\mathrm{tPA}^{-1-}$ mice were infused with $120 \mathrm{ng} / \mathrm{ml} \mathrm{tPA}$ before KA injection). When NF-L-tPA mice were coinjected with tPA and KA, increased neuronal death was observed by $12 \mathrm{hr}(\sim 50 \%$ of the pyramidal neurons were dead) compared with those injected with KA alone $(\sim 10 \%)$. Microglia in these mice were as activated morphologically as their counterparts in mice injected with KA alone; however, higher numbers of microglial cells were detectable after the tPA-KA coinjection.

Furthermore, the possibility that the low levels of tPA made may cause a slower rate of degeneration in NF-L-tPA mice was 
ruled out for three reasons: (1) many NF-L-tPA neurons die at earlier time points, (2) NF-L-tPA neurons eventually degenerate to the same extent as Fms-tPA neurons, and (3) Fms-tPA neurons die faster with apparently similar amounts of tPA made.

We conclude that the microglial contribution in the early time points after KA excitotoxicity is very important, if not key, to differentiating between neuronal death and survival.

The combined delay in neuronal death and early microglial activation in NF-L-tPA mice suggests that early neuronal release of tPA may be a signal to activate microglia. However, these microglia are not able to induce their own proliferation and become neurotoxic because they lack the tPA-mediated paracrine/autocrine activation. Therefore, there is a containment of the neurodegeneration occurring in the NF-L-tPA mice, at least in the first few days after excitotoxicity. Eventually, neighboring injured neurons may secrete sufficient tPA to activate microglia to become neurotoxic.

In the Fms-tPA mice, although neurons are not expressing tPA, nearby microglia can sense the initial insult sustained by these neurons because of KA. We do not know what this signal may be, although injured neurons secrete many factors. The microglia then become activated and secrete sufficient amounts of tPA to both recruit microglia to the vicinity of injury and cleave neuronally secreted plasminogen into plasmin. One point of contention in this scenario is that the mechanism by which neurons and microglia regulate their tPA synthesis and release after KA insult may be different, and in our case, the transgenes are not under endogenous tPA promoter controls.

We have hypothesized that a high concentration of tPA, acting along with a yet unidentified costimulating molecule, activates microglia after injury (Siao and Tsirka, 2002). Because it appears that the ability of NF-L-tPA microglia to become activated is not diminished, we presume that the threshold concentration has been met in vivo (possibly by tPA secreted from nearby injured neurons). It is still possible that the transgenic microglia from either genotype are susceptible to tPA-mediated activation to a different degree than wild-type microglia, because they may be exposed to higher levels of constitutive tPA because of the transgene. We have begun to characterize the population of microglia from these transgenic mice.

The results from NF-L-tPA mice imply that either there are subpopulations of microglia, which play different roles after induction of excitotoxicity, or microglia can play dual roles in injury. There is controversy concerning the first point (Streit et al., 1999). An examination of the cells around the injury site in wildtype and transgenic mice suggests that perineuronal microglia behave differently from distal microglia. Using a marker that recognizes primarily activated microglia (5D4), we noted that perineuronal microglia do not change shape immediately. Morphologically full activation and recruitment is observed with the more distal microglia from the corpus callosum or stratum radiatum. One example of this observation is seen in Figure 6 (NF-LtPA at $12 \mathrm{hr}$ after KA injection). In addition, even in wild-type animals the microglia that become activated at $2 \mathrm{~d}$ after KA injection are farther from the CA1 neuronal layer (Fig. 6), a result that is in agreement with the findings of Andersson et al. (1991). Perineuronal microglia (in conjunction with injured neurons) may act as amplifiers to signal to, or recruit, remote microglia, using chemotactic gradients such as monocyte chemoattractant protein-1 (Galasso et al., 2000; Flugel et al., 2001). These microglia may not present as fully activated cells morphologically, because they have not had time to change shape. However, the morphological changes observed are not the representation of early signs of activation. Changes in gene expression occur first, resulting in the secretion of the factors (i.e., TNF- $\alpha$, NO, proteases) that possibly enable activated microglia to display neurotoxic properties. An output of these gene expression changes is the morphological events observed, and they reach maximal stage at day 5 after KA injection (Andersson et al., 1991). Therefore, it appears possible that activating rather than activated microglia are neurotoxic. Eventually, all microglia become fully activated and cluster around dying and dead neurons.

It is becoming apparent that microglia can play both neuroprotective and neurotoxic roles in the CNS after injury. The NFL-tPA transgenic mice show less severe neurodegeneration but more activated microglia immediately after excitotoxicity, although only neurons secrete tPA. This observation suggests that tPA released soon after KA injection by injured neurons acts as a signal to activate microglia, possibly to contain apoptotic cells and cell debris. Therefore, the ability of these microglia to limit initial neuronal injury or death may be significant.

NF-L-tPA neurons continue to die days after the KA injection, concordant with an increase in microglial activation, suggesting that activated microglia can also be neurotoxic. This observation, along with the more severe early neurodegeneration seen in FmstPA mice after KA injury, shows that activated microglia continue to recruit and activate other microglia and secrete neurotoxic molecules. The hyperactivated microglia can be detrimental to neighboring neurons, which die a secondary apoptotic death (after the initial death resulting from the KA insult). Our results suggest that there is a window of opportunity for clinically relevant microglial activation blockers to prevent such a bystander death. Given that there are small-molecular-weight (and possibly able to cross the $\mathrm{BBB}$ ) inhibitors that interact with $\mathrm{APA}$, one could envision using those to inhibit the neurotoxicity-inducing microglial activation. From these data, we conclude that $\mathrm{PA}$ and microglia are beneficial within moderate amounts and cell numbers or within a certain time frame after injury, but as the numbers of activated microglial cells increase, they become neurotoxic.

\section{References}

Akassoglou K, Probert L, Kontogeorgos G, Kollias G (1997) Astrocytespecific but not neuron-specific transmembrane TNF triggers inflammation and degeneration in the central nervous system of transgenic mice. J Immunol 158:438-445.

Akiyama H, Nishimura T, Kondo H, Ikeda K, Hayashi Y, McGeer P (1994) Expression of the receptor for macrophage colony stimulating factor by brain microglia and its upregulation in brains of patients with Alzheimer's disease and amyotrophic lateral sclerosis. Brain Res 639:171-174.

Andersson P, Perry V, Gordon S (1991) The kinetics and morphological characteristics of the macrophage-microglial response to kainic acidinduced neuronal degeneration. Neuroscience 42:201-214.

Banati R, Graeber M (1994) Surveillance, intervention and cytotoxicity: is there a protective role of microglia? Dev Neurosci 16:114-127.

Baranes D, Lederfein D, Huang YY, Chen M, Bailey C, Kandel E (1998) Tissue plasminogen activator contributes to the late phase of LTP and to synaptic growth in the hippocampal mossy fiber pathway. Neuron 21:813-825.

Bertolottoa A, Agrestib C, Castelloa A, Manzardoa E, Riccio A (1998) 5D4 keratan sulfate epitope identifies a subset of ramified microglia in normal central nervous system parenchyma. J Neuroimmunol 85:69-77.

Blevins G, Fedoroff S (1995) Microglia in colony stimulating factor 1-deficient op/op mice. J Neurosci Res 40:535-544.

Cardone M, Salvesen G, Widmann C, Johnson G, Frisch S (1997) The regulation of anoikis: MEKK-1 activation requires cleavage by caspases. Cell 90:315-323.

Chao CC, Hu S (1994) Tumor necrosis factor-alpha potentiates glutamate neurotoxicity in human fetal brain cell cultures. Dev Neurosci 16:172-179.

Chen Z-L, Strickland S (1997) Neuronal death in the hippocampus is promoted by plasmin-catalyzed degradation of laminin. Cell 91:917-925. 
Cuzner M, Gveric D, Strand C, Loughlin A, Paemen L, Opdenakker G, Newcombe J (1996) The expression of tissue-type plasminogen activator, matrix metalloproteases and endogenous inhibitors in the central nervous system in multiple sclerosis: comparison of stages in lesion evolution. J Neuropathol Exp Neurol 55:1194-1204.

Dirnagl U, Iadecola C, Moskowitc M (1999) Pathobiology of ischaemic stroke: an integrated view. Trends Neurosci 22:391-397.

Flavin M, Zhao G, Ho L (2000) Microglial tissue plasminogen activator (tPA) triggers neuronal apoptosis in vitro. Glia 29:347-354.

Flugel A, Hager G, Horvat A, Spitzer C, Singer G, Graeber M, Kreutzberg G, Schwaiger F (2001) Neuronal MCP-1 expression in response to remote nerve injury. J Cereb Blood Flow Metab 21:69-76.

Frey U, Müller M, Kuhl D (1996) A different form of long-lasting potentiation revealed in tissue plasminogen activator mutant mice. J Neurosci 16:2057-2063.

Galasso J, Liu Y, Szaflarski J, Warren J, Silverstein F (2000) Monocyte chemoattractant protein-1 is a mediator of acute excitotoxic injury in neonatal rat brain. Neuroscience 101:737-744.

Gualandris A, Jones T, Strickland S, Tsirka S (1996) Membrane depolarization induces the $\mathrm{Ca}^{2+}$-dependent release of tissue plasminogen activator. J Neurosci 16:2220-2225.

Hastings G, Coleman T, Haudenschild C, Stefansson S, Smith E, Barthlow R, Cherry S, Sandkvist M, Lawrence D (1997) Neuroserpin, a brainassociated inhibitor of tissue plasminogen activator, is localized primarily in neurons. J Biol Chem 272:33062-33067.

Jacovina AT, Zhong F, Khazanova E, Lev E, Deora AB, Hajjar KA (2001) Neuritogenesis and the nerve growth factor-induced differentiation of PC-12 cells requires annexin II-mediated plasmin generation. J Biol Chem 276:49350-49358.

Jander S, Stoll G (1996) Downregulation of microglial keratan sulfate proteoglycans coincident with lymphomonocytic infiltration of the rat central nervous system. Am J Pathol 148:71-78.

Jones L, Tuszynski M (2002) Spinal cord injury elicits expression of keratan sulfate proteoglycans by macrophages, reactive microglia, and oligodendrocyte progenitors. J Neurosci 22:4611-4624.

Kreutzberg G (1995) Microglia, the first line of defense in brain pathologies. Arzneimittelforschung 45:357-360.

Kreutzberg G (1996) Microglia: a sensor for pathological events in the CNS. Trends Neurosci 19:312-318.

Kritzer M (2000) Effects of acute and chronic gonadectomy on the catecholamine innervation of the cerebral cortex in adult male rats: insensitivity of axons immunoreactive for dopamine-B-hydroxylase to gonadal steroids, and differential sensitivity of axons immunoreactive for tyrosine hydroxylase to ovarian and testicular hormones. J Comp Neurol 427:617-633.

Krueger SR, Ghisu GP, Cinelli P, Gschwend TP, Osterwalder T, Wolfer DP, Sonderegger P (1997) Expression of neuroserpin, an inhibitor of tissue plasminogen activator, in the developing and adult nervous system of the mouse. J Neurosci 17:8984-8996.

Lawson L, Perry V, Dri P, Gordon S (1990) Heterogeneity in the distribution and morphology of microglia in the normal adult mouse brain. Neuroscience 39:151-170.

Madani R, Hulo S, Toni N, Madani H, Steimer T, Muller D, Vassalli JD (1999) Enhanced hippocampal long-term potentiation and learning by increased neuronal expression of tissue-type plasminogen activator in transgenic mice. EMBO J 18:3007-3012.

Mattson M, Cheng B, Davis D, Bryant K, Lieberburg I, Rydel R (1992) $\beta$-amyloid peptides destabilize calcium homeostasis and render human cortical neurons vulnerable to excitotoxicity. J Neurosci 12:376-389.
McMillian M, Vainio P, Tuominen R (1997) Role of protein kinase C in microglia-induced neurotoxicity in mesencephalic cultures. J Neuropathol Exp Neurol 56:301-307.

Noda M, Nakanishi H, Akaike N (1999) Glutamate release from microglia via glutamate transporter is enhanced by amyloid-beta peptide. Neuroscience 92:1465-1474.

Olney J (1986) Inciting excitotoxic cytoside among central neurons. Adv Exp Med Biol 203:631-645.

Parmer RJ, Mahata M, Mahata S, Sebald MT, O’Connor DT, Miles LA (1997) Tissue plasminogen activator ( $t-P A)$ is targeted to the regulated secretory pathway. Catecholamine storage vesicles as a reservoir for the rapid release of t-PA. J Biol Chem 272:1976-1982.

Pitt D, Werner P, Raine C (2000) Glutamate excitotoxicity in a model of multiple sclerosis. Nat Med 6:67-70.

Raivich G, Haas S, Werner A, Klein M, Kloss C, Kreutzberg G (1998) Regulation of MCSF receptors on microglia in the normal and injured mouse central nervous system-a quantitative immunofluorescence study using confocal laser microscopy. J Comp Neurol 395:342-358.

Rickles R, Darrow A, Strickland S (1988) Molecular cloning of cDNA to mouse tissue plasminogen activator mRNA and its expression during F9 teratocarcinoma cell differentiation. J Biol Chem 263:1563-1569.

Rogove A, Tsirka S (1998) Neurotoxic responses by microglia elicited by excitotoxic injury in the mouse hippocampus. Curr Biol 8:19-25.

Rogove A, Siao C, Keyt B, Strickland S, Tsirka S (1999) Activation of microglia reveals a non-proteolytic cytokine function for tissue plasminogen activator in the central nervous system. J Cell Sci 112:4007-4016.

Sallés F, Strickland S (2002) Localization and regulation of the tissue plasminogen activator-plasmin system in the hippocampus. J Neurosci 22:2125-2134.

Sappino A, Madani R, Huarte J, Belin D, Kiss J, Wohlwend A, Vassalli J (1993) Extracellular proteolysis in the adult murine brain. J Clin Invest 92:679-685.

Schlaepfer W, Bruce J (1990) Simultaneous up-regulation of neurofilament proteins during the postnatal development of the rat nervous system. J Neurosci Res 25:39-49.

Seeds N, Williams B, Bickford P (1995) Tissue plasminogen activator induction in Purkinje neurons after cerebellar motor learning. Science 270:1992-1994.

Siao C-J, Tsirka S (2002) Tissue plasminogen activator mediates microglial activation via its finger domain through annexin II. J Neurosci 22:3352-3358.

Streit WJ, Walter SA, Pennell NA (1999) Reactive microgliosis. Prog Neurobiol 57:563-581.

Thanos S, Mey J, Wild M (1993) Treatment of the adult retina with microglia-suppressing factors retards axotomy-induced neuronal degradation and enhances axonal regeneration in vivo and in vitro. J Neurosci 13:455-466.

Tsirka S, Gualandris A, Amaral D, Strickland S (1995) Excitotoxin induced neuronal degeneration and seizure are mediated by tissue-type plasminogen activator. Nature 377:340-344.

Tsirka S, Rogove A, Bugge T, Degen J, Strickland S (1997) An extracellular proteolytic cascade promotes neuronal degeneration in the mouse hippocampus. J Neurosci 17:543-552.

Wu YP, Siao CJ, Lu W, Sung TC, Frohman M, Milev P, Bugge T, Degen J, Levine J, Margolis R, Tsirka S (2000) The tPA/plasmin extracellular proteolytic system regulates seizure-induced hippocampal mossy fiber outgrowth through a proteoglycan substrate. J Cell Biol 148:1295-1304. 